\title{
ON EXPERIMENTAL OBSERVATIONS OF SEISMOELECTRIC EFFECT AT GAS CONDENSATE DEPOSIT IN EARTH'S ELECTROMAGNETIC AND SEISMIC NOISES
}

\author{
* G. Ya. Shaidurov ${ }^{1}$, V. S. Potylitsyn ${ }^{1}$, D. S. Kudinov ${ }^{1}$, O. A. Maikov ${ }^{1}$ \\ ${ }^{1}$ Siberian Federal University, Russia
}

*Corresponding Author, Received: 09 May. 2018， Revised: 21 Aug. 2018, Accepted: 25 Sept. 2018

\begin{abstract}
The main aim of this work is physical interpretation and experimental verification of the method based on a recording of seismoelectric effect in natural Earth's seismic and electromagnetic fields. In this case, a deposit is described by a model of the flat condenser in which ion-conducting medium (deposit waters) serve as plates and deposited hydrocarbons serve as a dielectric layer. The considered frequencies of both types of fields are in the range of $0.1-20 \mathrm{~Hz}$. The calculated curve of electric field distribution on Earth's surface generated by deposit seam induced by natural electric and seismic fields of the Earth's crust is given. Experimental data of observations of seismoelectric effect in passive fields are presented obtained in 20142017 at Bystryansky gas condensate field (Krasnoyarsk Krai, Minusinsk district) by the recording of passive electric and seismic fields as well as measurement of their cross-correlation.
\end{abstract}

Keywords: Deposit, Hydrocarbons, Electric field, Seismic, Noises

\section{INTRODUCTION}

Seismoelectric effect (SEE) was described for the first time by Ivanov [1, 2], further on this issue was discussed in [3, 4], at present in Russia articles are available presenting theoretical models and results of laboratory and field observations [5, 6].

In foreign publications this issue was discussed by Berg-Vishnyakov [7-9] during combined processing of secondary electric and seismic signals from appropriate sensors; they also protected the US Patent: Method of Seismo-Electromagnetic Detecting of Hydrocarbon Deposit [7]. The data are available on observation of this effect on hydrocarbon deposits of the Gulf of Mexico and Arctic seas of Russian shelf [8]. The authors of these works state that the new method increases a chance to detect productive deposits by 4 times in comparison with conventional seismic exploration. The described results are obtained upon application of artificial sources of seismic and electromagnetic fields.

In 2014-2017 we observed the SEE in passive fields at Minusinsk gas condensate deposit $[10,11]$ (Khakassia, Russia).

An obvious advantage of the passive method is the simplicity of its instrumentational implementation and its costs in comparison with the active method, as well as possible to perform exploration in mountain forest areas where it is difficult to apply conventional exploration activities due to complex relief.

The passive method is characterized by certain disadvantages. For instance, its application will be accompanied by signal accumulation and taking into account that the involved frequencies are sufficiently low $(0.1-20 \mathrm{~Hz})$, the required time can reach 1-15 min; in addition, measurement of electric constituent of the Earth's horizontal field in winter upon ground freezing to grounded electric dipole will be impossible, and in this case it will be required to use specialized low-frequency antennas.

It should be mentioned that for field applications the highest interest will be attracted only by methods not based on additional sources of electromagnetic fields, that is, without extra costs.

Therefore, application of SSE upon hydrocarbon exploration will be restricted by two methods. The first method is semi-active (conventional exploration with simultaneous recording of electromagnetic fields). The second method is a completely passive method with a recording of both seismic and electric fields.

This work discusses the passive seismoelectric method of hydrocarbon exploration and analyzes experimental observations on actual gas condensate deposits in the Earth's passive electromagnetic and seismic fields.

\section{METHODS}

Let us estimate seismoelectric effect by solving the inverse problem: calculation of electric moment of equivalent dipole which is located in the seam center and creates electric field on the Earth's surface, in this case, a deposit is simulated as flat condenser charged with electric field $E_{0}$, surface area of its plates equals to that of the deposit. 
The plates of equivalent condenser oscillate under the action of natural seismic perturbations varying the moment of the equivalent dipole, and an electric field is generated in host medium which can be recorded on the Earth's surface.

Figure 1 illustrates a schematic view of the equivalent condenser (physical model) depicting hydrocarbon seam with linear dimensions of $L \times L$, width d, and appropriate dielectric permeability $\varepsilon_{2}$, electric conductivity $\sigma_{2}=0$, located at the depth $\mathrm{h}$. This seam is influenced by the Earth's static field $E_{0}$.

In order to analyze the intensity of electric field $E_{x}$ on the Earth's surface in the point $\mathrm{N}$, the oscillations of condenser plates under the action of seismic oscillations acting on hydrocarbon seam were taken into account. We have already developed equations for $E_{x}$ with consideration for hydrocarbon seam, and in this work, we will apply the obtained final equations [11]:

$E_{X}=\frac{\varepsilon_{2} \tau_{1}^{2} E_{0} \Delta P}{4 \pi \varepsilon_{1} \rho} \cdot \Phi$

where $\varepsilon_{1}$ is the dielectric permeability of host medium, $\varepsilon_{2}$ is the dielectric permeability of seam, $\tau_{1}$ is the time of pressure pulse, $\Delta P$ is the pressure amplitude of the seismic wave, $\rho$ is the medium density, $\Phi$ is the geometrical coefficient:

$$
\Phi=\frac{h d x d y}{d} \cdot \sum_{i=1}^{n} \sum_{i=1}^{n} \frac{(x-i d x)}{\left[\left(x-x_{i}\right)^{2}+((i-1) d y)^{2}+h^{2}\right]^{\frac{5}{2}}} .
$$

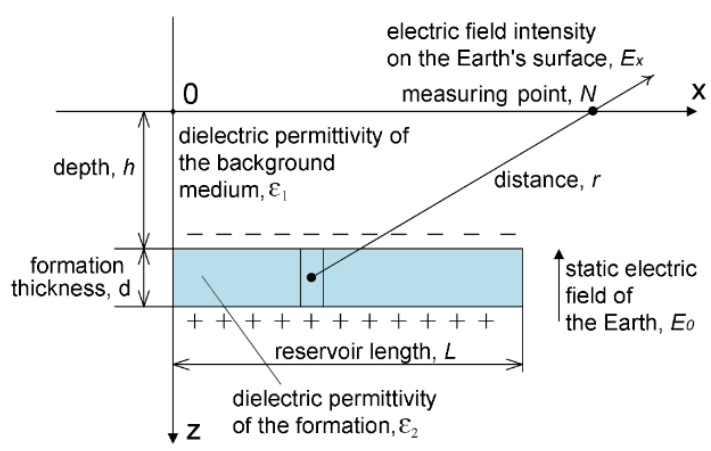

Fig. 1 Hydrocarbon productive seam

In order to analyze the pressure, $\Delta \mathrm{P}$ let us apply the experimental data in [11]. The output voltage of GS-20DX geophone upon field tests was $U=5 \cdot 10^{-6}$ $V$ with the conversion index $K=0.28 \mathrm{~V} /(\mathrm{cm} / \mathrm{s})$. The medium density was considered to be $\rho_{1}=1.8 \cdot 10^{3}$ $\mathrm{kg} / \mathrm{m}^{3}$, and the velocity of elastic oscillations was $V=4000 \mathrm{~m} / \mathrm{s}$. Therefore:
$\Delta P=\frac{U \cdot \rho_{1} \cdot V}{K}=1.28 P a$

With the aim of comparison, in 2017 seismic and electric noises were measured at Bystryansky gas condensate deposit (Krasnoyarsk krai, Minusink district) in the locations highlighted in the map by passive method (without excitation of the field by natural seismic and electromagnetic field). The depth of gas seam for this deposit was about $2000 \mathrm{~m}$.

Similar results were obtained previously in 2015-2016.

Figure 2 illustrates the experimental layout. The measurements were carried out using an SGD-SET seismostation, signals from seismic cables with connected SGD-SET/FU receivers and 200m electric dipole installed at the center of the cables were supplied to the station input. Non-polarized VITR electrodes were used as electrodes. The output of the electric dipole was connected to free receiving channels of the seismostation via specialized receiver with amplifier and bandwidth $(0.1-20 \mathrm{~Hz})$ and suppression of industrial noises of at least $60 \mathrm{~dB}$, the output of seismic cable was connected to main channels of the seismostation. Herewith, mean root square electric noise was 100 $\mu \mathrm{V}$ corresponding to the field intensity of $0.5 \mu \mathrm{V} / \mathrm{m}$.

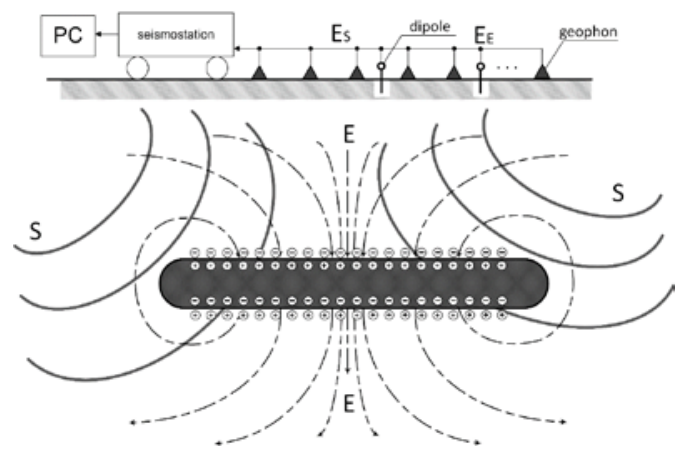

Fig. 2 Experimental layout

The receiver was based on INA128 (Texas Instruments) instrumental band differential amplifier. This circuit provided output resistance of about $3 \mathrm{M} \Omega$, suppression of in-phase signal up to $120 \mathrm{~dB}$ and capability to control the coefficient of amplification by one potentiometer, which facilitated wide dynamic range and amplification of input signal from 1 to 10000 . The required amplification was set by a digital potentiometer with a microcontroller. In order to suppress industrial noise of $50 \mathrm{~Hz}$, a band exclusion filter of the 4th order was installed between the cascades.

It was established in 2015-2016 that the most suitable frequency range for electric field recording was $0.1-20 \mathrm{~Hz}$. This frequency band was isolated 
by a MAX293 switched-capacitor filter. This microcircuit is a filter of lower frequencies with adjustable cut-off frequency adjusted by variation of the clock frequency. The lower limit of $0.1 \mathrm{~Hz}$ was provided by the filter of upper frequencies of the second order and eliminated drift of electrode zero. The receiver was controlled by STM32F103 microcontroller based on Cortex-M3.

This microcontroller controlled the following properties of the receiver: amplification and cut-off frequencies. In addition, it facilitated analog to digital conversion of signals and transmission of appropriate data to PC using USB interface.

Flowchart and appearance of the developed receiver circuits are illustrated in Figs. 3 and 4.

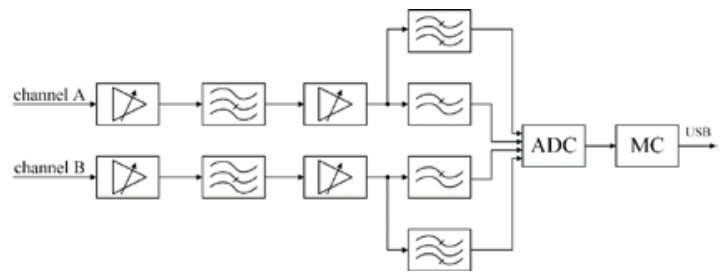

Fig. 3 Experimental layout

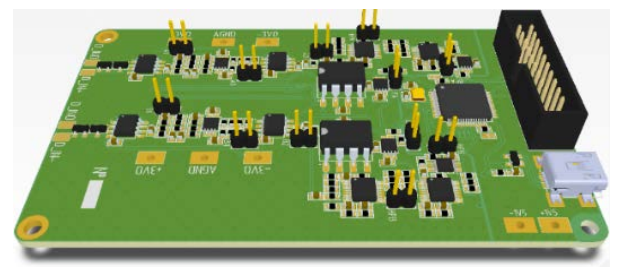

Fig. 4 Experimental layout

The algorithm of calculation of the coefficient of cross-correlation (CCC) was carried out by subsequent processing in Matlab environment. CCC was calculated as follows:

$$
R_{E S}(n)=\frac{1}{N} \cdot \sum_{i=1}^{N} \bar{E}_{i} \cdot \bar{S}_{i-n},
$$

where $\bar{E}_{i}$ and $\bar{S}_{i}$ were the disperse normalized signals from electric dipole and receiver, respectively; $N$ was the number of signal count, $i$ was the number of appropriate signal count, $n$ was the positive integer.

The software interface is illustrated in Fig. 5. This software makes it possible to plot time realization of signals, their spectra, crosscorrelation function, to carry out digital filtration of signals. In addition, it displays measured CCC in the point of measurement. With the aim of further processing, the software stores all recorded signals in flash memory.

Thus, it is possible in real time to estimate measurement results and adjust schedules of field activities using the profile. Post processing includes plotting of CCC profile, comparison of measurement data with the results obtained by other methods, such as seismic exploration and others.

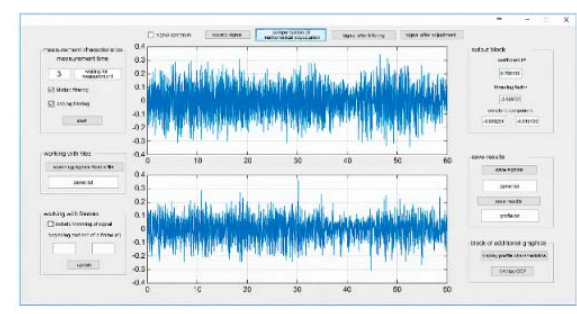

Fig. 5 Software interface for a data processing

Coordinates of measurement points of CCC were determined using GPS/GLONASS. Subsequently, the function of cross-correlation between electric and seismic noise signals was calculated by algorithm 3 .

Figure 6 illustrates the geological map of Bystryansky gas condensate field with highlighted exploration wells and observation profile of CCC $\mathrm{R}_{\mathrm{ES}}(0)$ in various seasons.

Green and blue lines are the measurement profiles in 2014-2015 and 2016, respectively. Black circles denote exploration wells; near well 5$\mathrm{P}$ gas was released in the form of jet fire.

Dashed and solid brown line denote deposit contour obtained by seismic exploration and exploring drilling at various depth.

Performance of operation at one and the same profile in different years is stipulated by necessity to provide repeatability at different conditions of observations, such as weather, daytime and others. In this case, passive noise electromagnetic and seismic field is assumed, statistic data processing is required for observation points.

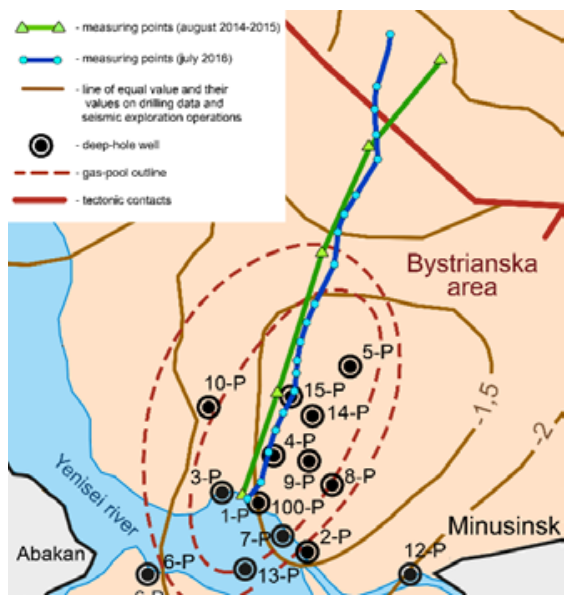

Fig. 6 Geological map of Bystryansky gas condensate deposit (Krasnoyarsk krai, Minusinsk district). 


\section{RESULTS AND DISCUSSION}

Figure 7 (a) illustrates the results of profiling by SEE for various points of observations in the years 2014-2015, Fig. 7 (b) - the calculation results by Eq.(1) with consideration for Eqs. (2) and (3) for the module of electric field on the Earth's surface as a function of distance to abnormality center with the seam dimensions of $L=1000 \mathrm{~m}, d=100 \mathrm{~m}$ and the seam depth of $h=2000 \mathrm{~m}$. The seam density is $\rho=0.86 \cdot 10^{3} \mathrm{~kg} / \mathrm{m}^{3}$ and its relative dielectric permeability is $\varepsilon_{2}=2.18$, the relative dielectric permeability of the host medium is $\varepsilon_{1}=10$. The pressure amplitude of elastic oscillations is $\Delta P=1.28 \mathrm{~Pa}$ at $\tau_{1}=1 \mathrm{~s}$, which corresponds to the frequency band of $1 \mathrm{~Hz}$.
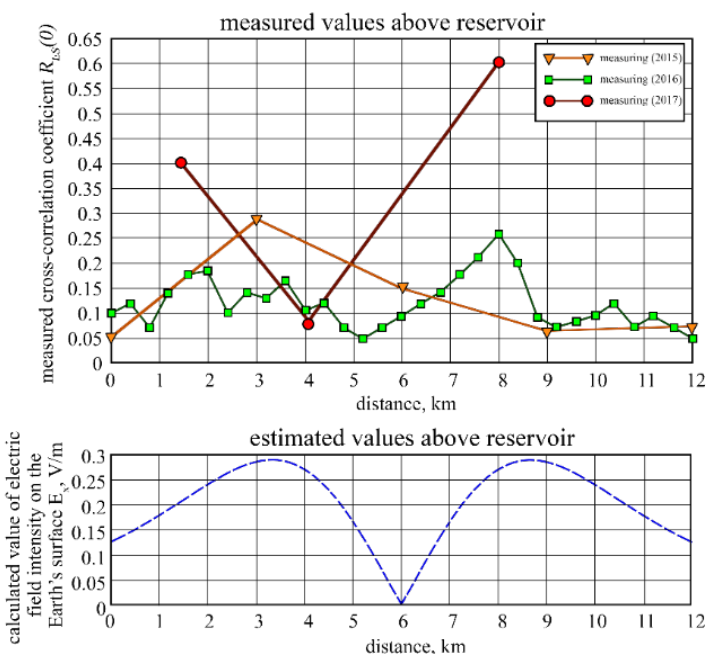

Fig. 7 (a) Profiling by SEE method; (b) Electric field distribution over a model of the productive seam

As can be seen, the abnormal area is highlighted by CCC $R_{E S}(0)$ by 3-5 times higher than that of the normal area. This result was qualitatively reproduced in three years except for certain scatter of amplitude. As can be seen in the plots, the amplitude of $R_{E S}(0)$ varies from year to year but the pattern of the curves is reproduced. We believe that this can be related both to different weather conditions and with the different seismic situation above the deposit requiring for additional observations. The calculated curve has two maxima of $E_{x}$ module at the seam edges. Analysis of the plots shows that the most distinct is the seam boundaries, which corresponds to experimental data.

As follows from Fig. 7, the CCC maximum in different years is observed at the distances of 2-3 $\mathrm{km}$ and $8 \mathrm{~km}$, and the minimum - at $4-5 \mathrm{~km}$. In this case, the results are characterized by certain instability. We believe that this can be attributed to short observation time which equaled for these measurements to $60 \mathrm{~s}$. Increase in time to 5-10 min will make it possible to average the influence of local noises of both seismic and electromagnetic origin, as well as to increase the signal to noise ratio by means of signal accumulation.

These CCC values obtained in 2017 are in fact by two times higher in amplitude than those of other years but taking into account that these measurements were performed only at three profile points, this does not provide a complete pattern. Thus, a more detailed analysis of profile is required, as in 2016.

Figure 8 (a, b) illustrates plots of data processing obtained both in abnormality area at the deposited edge (Fig. 8b) and in the normal field (Fig. 8a). In this case, $\tau_{\mathrm{E}}$ is the start of the time interval of the grounded electric dipole, $\tau_{\mathrm{S}}$ is the start of the time interval of one of the receivers, $R_{E S}(0)$ is the maximum of the cross-correlation function.
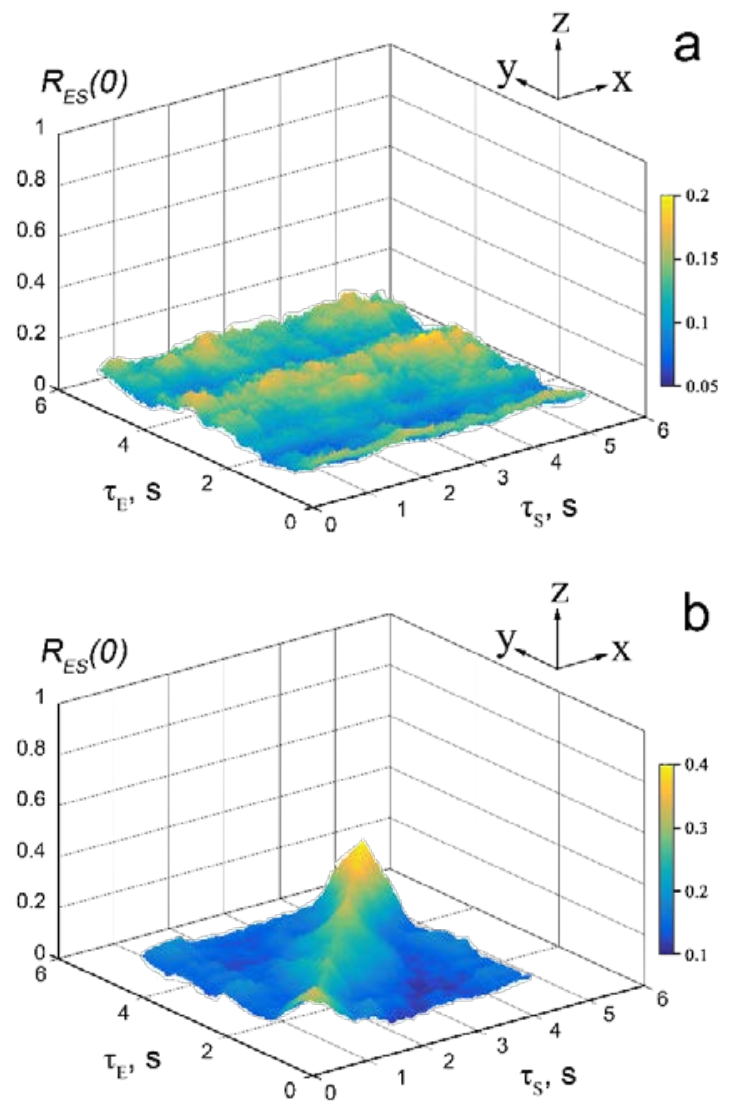

Fig. 8 CCC maximum as a function of time interval shift (a) - normal field (without deposit), (b) - at the edge of abnormality at Bystryansky gas condensate deposit

For further data processing, the time interval was 1 second, data were acquired 10 times with subsequent averaging.

The plots demonstrate that in the normal field (without deposit) it is possible to observe noise pattern not exceeding $\mathrm{R}_{\mathrm{ES}}(0)=0.2$, which evidences the absence of cross-correlation between noise seismic and electric fields. Figure 4(b) 
illustrates "ridge" in the form of CCC maximum at equal time delays of electromagnetic and seismic fields, which is determined by CCC computations when one of the signals reaches the surface earlier and is artificially shifted in order to obtain the maximum. Oscillations from $\mathrm{R}_{\mathrm{ES}}(0)=0.2$ to $\mathrm{R}_{\mathrm{ES}}(0)$ $=0.4$ are stipulated by low time interval equaling to $1 \mathrm{~s}$. As mentioned above, increase in acquisition frequency will permit to average these oscillations and to obtain more stable values of $\mathrm{R}_{\mathrm{ES}}(0)$. Therefore, it is necessary to determine the experimentally optimum time of acquisition and time interval.

It is obvious that physical interpretation of the obtained results requires for additional experimental and theoretical analysis beyond the scope of this article.

\section{CONCLUSION}

Experimental observations of existing gas condensate deposit in the Earth's passive electromagnetic and seismic fields were analyzed, theoretical data acquired at Bystryansky gas condensate field in different years were compared.

The obtained results confirm data acquired by other researchers [5, 9] on the basis of active electromagnetic and seismic field sources.

Gas condensate deposit is satisfactorily described by a model of seam deposit, which is confirmed by experimental data obtained in different years. Characteristic CCC increase is observed at the deposit edges.

Concerning the application of this method, it is possible to state that in future it would permit to detect deposits, that is, to fix contours of abnormality, further, identification will require for additional researches using conventional methods.

Further schedule assumes measurements at other hydrocarbon deposits, petroleum in particular, by geophysical crews and subsequent comparison of the obtained results.

\section{ACKNOWLEDGMENTS}

The authors thank the Siberian Federal University for providing the infrastructure with the implementation of this project. This work has been done with the support of Council on grants of the President of the Russian Federation (scholarships of the President of the Russian Federation to young scientists and graduate students № 904.2018.1) and a grant of the Russian Science Foundation (project No. 18-79-00137.

\section{REFERENCES}

1. Ivanov G., Earth Layers Charging Electric
Effect When Affected by Elastic Waves, Reports of the USSR Academy of Sciences, No. 1, 1939, p. 24.

2. Ivanov G., Seismoelectric Effect of the II Kind, USSR Academy of Sciences Review, Geology and Geophysics, No. 5, 1940.

3. Antsiferov, M.S., Electroseismic Effect. The Application of Seismoelectrical Methods in Mining, Moscow, Nauka, 1964.

4. Gubatenko W.P., Svetov B.S., Moskovski I.G., Electromagnetic Field of Mechano-Electrical Origin in Porous Waterlogged Rock, Calculations used in Horizontally Layered Earth, Physics of the Earth. Vol. 2, No. 2, 2002, pp. 34-50.

5. Melnikov V.P., Lisov V.I., Bryukhovetski O.S, Bobrovnikov L.Z., Innovative Geophysics: Binary Technologies of Direct Exploration. Part 1, Petroleum Engineer, IDS Drilling, No. 1, 2010, pp. 47-51.

6. Dobrynin S.I., Golovin S.V., Bobrovnikov L.Z., Melaev D.V., Innovative Technology of Hydrocarbon Deposits Assessment and Monitoring in Oil and Gas Develop Reserves, Petroleum Engineer. Moscow: IDS Dilling, No. 2, 2012, pp. 24-29.

7. Vishnyakov-Berg E., Panyaev V.P., Yanevichet M.Y., Theory and Method Development Used for Oil and Gas Direct Exploration, Production geological Association Funds «Sevmorgeo», Arctic Geology Development Institute, 1981, p. 176.

8. Berg E., Method of Seismo-Electromagnetic Detecting of Hydrocarbon Deposit, US Patent No. 7330790. October 2005

9. Berg-Vishnyakov A.E., Technology and Experience of Application of Electromagnetic Transient Buildup Affected by Seismic Radiation (TBSR) in the Search for Raw Hydrocarbon Deposits in conditions of shelf, Materials of the X-th international geophysical scientific-practical seminar «The application of modern electrical exploration technologies in prospecting of mineral deposits», St Petersburg, p. 35-39, 2012.

10. Shaidurov G.Ya., Kudinov D.S. Potylitsyn V.S., Kokhon`kova E.A., Shaidurov R.G., Pervye rezul taty nabliudeniia seismoelektricheskogo effect i vyzvannoi polyarizatsii na gazokondensatnom mestorozhdenii $\mathrm{v}$ estestvennykh shumovykh poliakh Zemli [First results of observation of seismoelectric effect and induced polarization at gas condensate deposit in natural noise of the Earth's fields], Pribory i sistemy razvedochnoi geofiziki [Instruments and systems of exploratory geophysics], InformGeofizServis, No. 1. 2016, pp. 81-88.

11. Shaidurov G.Ya., Romanova G.N., Kudinov 
D.S., Kokhonkova E.A., Potylitsyn V.S., Nabliudenie i fizicheskaia interpretatciia seismoelektricheskogo effekta na gazokondensatnom mestorozhdenii $\mathrm{v}$ elektromagnitnykh shumakh Zemli [Observation and physical interpretation of seismoelectric effect at gas condensate deposit in the Earth's electromagnetic fields], Pribory i sistemy razvedochnoi geofiziki [Instruments and systems of exploratory geophysics]. InformGeofizServis, 2017. No. 1, pp. 56-61.

Copyright (C) Int. J. of GEOMATE. All rights reserved, including the making of copies unless permission is obtained from the copyright proprietors. 